\title{
Detection of Glucoma and Diabetes through Image Processing and Machine Learning Approaches
}

\author{
Payal Bose ${ }^{1}$, Prof. Shawni Dutta ${ }^{2}$, Prof. Vishal Goyal ${ }^{3}$ and Prof. Samir K. Bandyopadhyay,"* \\ ${ }^{1}$ Research Scholar, Lincoln University College, Kota Bharu, Kelantan, Malaysia \\ ${ }^{2}$ Department of Computer Science, The Bhawanipur Education Society College, Kolkata, India \\ ${ }^{3}$ GLA University, Mathura-Delhi Road Mathura, Chaumuhan, Uttar Pradesh, India
}

${ }^{4}$ Distinguish Professor, Lincoln University College, Kota Bharu, Kelantan, Malaysia

* Corresponding author. (e-mail address:1954samir@gmail.com)

\begin{abstract}
In the last few decades, glaucoma became the second biggest leading cause of irreversible vision loss. Because of its asymptotic growth, it is not properly diagnosed until the relatively late stage. To stop the severe damage by glaucoma it is needed to detect glaucoma in its early stages. Surprisingly diabetes also be the greatest cause of glaucoma. In the modern era, artificial intelligence makes great progress in the medical image processing field. Image analysis based on machine learning gives a huge success in diagnosis glaucoma without any misdiagnosis. The aim of this proposed paper is to create an automated process that can detect glaucoma and diabetic retinopathy. Here various Machine Learning models are used and results of these methods are presented.
\end{abstract}

Keywords: Glaucoma, Diabetic Retinopathy, Convolution Neural Network (CNN), Vision Loss, Blindness, Machine Learning

\section{INTRODUCTION}

Glaucoma is a persistent condition that gradually damages the optic nerve of the eyes. It is the second largest cause of blindness in the world. It is a neurodegeneration disease and the ultimate conclusion is lost vision. When the intraocular pressure increases in the eye area, it starts to damage the optic nerves of the eyes gradually. Finally, when this damage is too high then the optic nerves stop sending visual images to the brain. And it causes total vision loss. To stop the severe damage, it is needed to detected glaucoma in the early stages. Glaucoma was diagnosed by four examinations: (1) measuring elevated intraocular pressure (IOP), (2) percentage damage to the optic disc by calculating the cup-to-disc ratio (CDR), (3) identifying decreased retinal nerve fiber layer (RNFL) thickness and (4) detection of characteristic visual field defects. The clinical test like optical coherence tomography (OCT) and visual field (VF) test display the indicators to diagnose glaucoma. But these conventional methods have higher chances of being misdiagnosed. In the modern era, artificial intelligence makes a great impact in the medical image processing field. Machine learning and image analysis give the greatest opportunity to detect glaucoma without 
being misdiagnosed. The main objective of this experiment is to create an automated glaucoma diagnosis system. So that the system can predict glaucoma at any stage very accurately and efficiently.

\section{RELATED WORKS}

To avoid the diagnosis the researchers, use artificial intelligence to detect glaucoma. They develop different machine learning models to create an automated glaucoma detection procedure. In today's world, machine learning became the leading tool for predicting and treating this severe disease. Researchers develop a machine learning predictive model that can select the five data features of the patients 1) visual field test,2) a retinal nerve fiber layer optical coherence tomography (RNFL OCT) test, 3) a general examination with 4) an intraocular pressure (IOP) measurement and 5) fundus photography. Finally, they used support vector machine (SVM), C5.0, random forest (RF), and XGboost algorithmsto test the predicted model [1].The researchers developed different prediction models based on deep learning techniques and use image data for prediction [2-6]. Also the traditional machine learning models areused for glaucoma prediction [7-9].Researchers comprehensively reviewed in their different articles about glaucoma, its types, cause, effect, and possible treatments. They used clinically as well as image processing, machine learning, and deep learning techniques to detect this disease effectively [10-11].

\section{GLAUCOMA}

In today's world glaucoma leads to the second largest disease for blindness. A single test cannot give the full information of glaucoma detection. The anatomy of the eye and the details about the optic nerve of the normal eye and glaucoma eyeare shown in figure 1(a,b,c) [12-13].

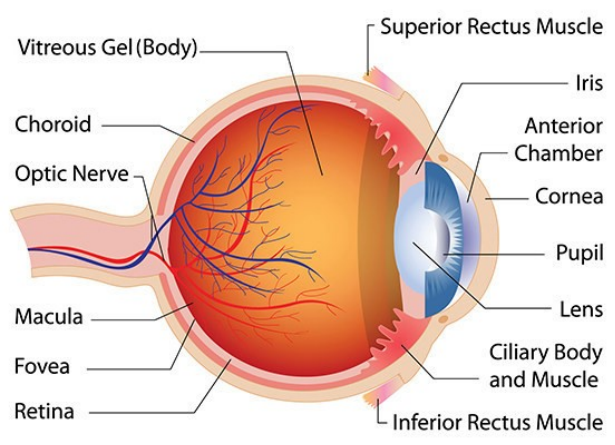

1(a) 


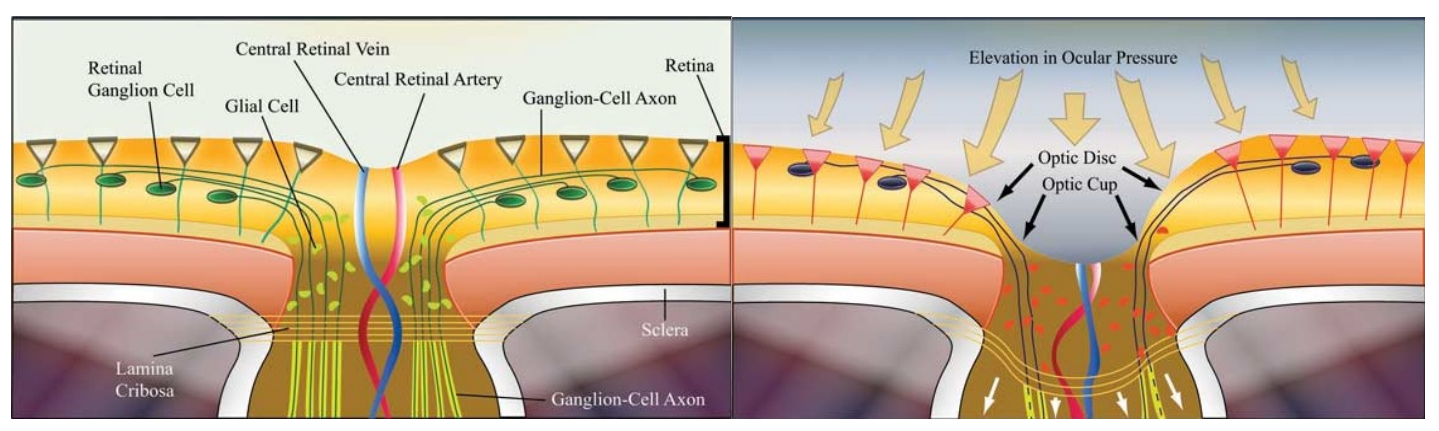

1(b)

$1(\mathrm{c})$

\section{Figure 1: a) Eye Anatomy, b) Healthy Eyes Optic Nerve, c) Glaucoma Eyes Optic Nerve}

\subsection{GLAUCOMA CAUSE AND RISK FACTORS}

The fluids flow through the eyes from a mesh-like channel is called aqueous humor [14]. Due to some unknown reason or the if eye fluid glands produce excessive fluids then this mesh-like channel gets blocked. This is the main cause of glaucoma. Some other reasons are available like chemical injury, blunt, blocked eye vessels, severe eye injuries, etc. The risk factors of glaucoma are given below,

- age over 40

- with near-sighted or far-sighted

- having poor vision

- having high diabetes

- Take certain drugs for bladder control or seizures, or some over-the-counter cold remedies

- Had an injury to one eye or both eyes

- The corneas are thinner than normal

- high blood pressure, heart disease, diabetes, or sickle cell anemia

- having high eye pressure

\subsection{DIABETIC AND GLAUCOMA}

Among the different risk factors of glaucoma, people with diabetes [15] are twice at risk of glaucoma than non-diabetic people. The most common glaucoma, Open-angle glaucoma is the affected diabetic patients in most of the cases or vice versa. High blood sugar also damages the tine vessels in the eyes. This damage can lead to a severe eye disease known as diabeticretinopathy. This disease blocks the natural drainage of fluid from the eyes and that causes glaucoma. Figure 2 shows the normal vs glaucoma vs diabetic glaucoma fundus images [16]. 


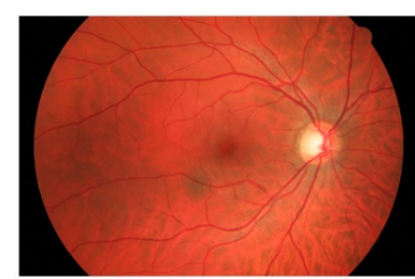

$\mathbf{2 a}$

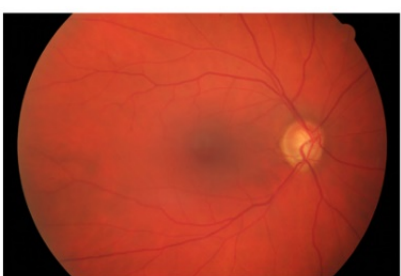

$\mathbf{2 b}$

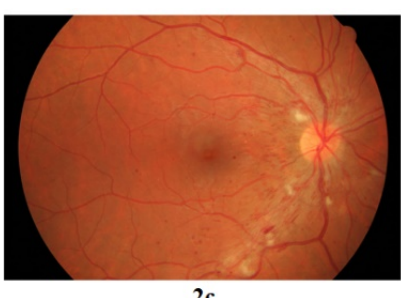

2c

Figure 2: a) Healthy Fundus Image, b) Glaucoma Fundus Image, c) Diabetic Glaucoma Fundus Image

\section{PROPOSED METHODOLOGICAL WORK}

The proposed methodology follows the three main steps. Figure 3 shows the main three steps of the proposed methodology.

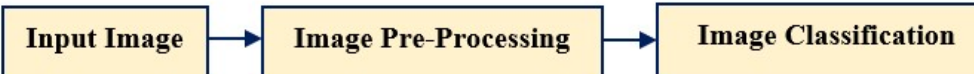

Figure 3: Main Steps of Proposed Methodology

\section{a. Input Image}

The input image database contains 15 image sets of healthy patients, diabetic retinopathy patients, and glaucoma patients [16]. All the input images are in RGB color space.

\section{b. Image Pre-Processing}

The pre-processing step is divided into four parts. Figure 4 shows the steps involve in the pre-processing.

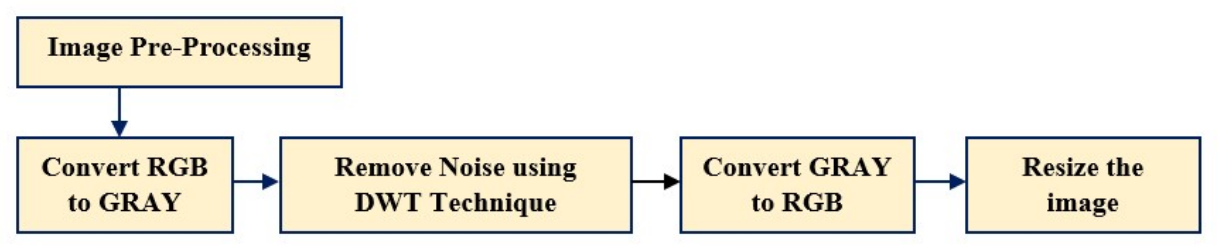

Figure 4: Steps of Pre-Processing

\section{c. Image Classification}

For image classification, different pre-trained convolution neural network models ResNet50 [17], VGG16 [18], VGG19 [19], InceptionV3 [20], and Xception [21]will be used. In order to get the best result to detect glaucoma, it is needed to fine-tune the required layers of the pre-trained models and also needed to adjust the epochs. The proposed algorithm of this experiment is given below.

\section{d. Algorithm}

Step1: Load the input RGB images.

Step2: Convert RGB to Gray Scale. 
Step3: Remove the noise of the image using Discrete Wavelet transformation techniques.

Step4: Convert the noise-free gray-scale images into RGB images.

Step5: Resize the images according to the network model's requirements.

Step6: Apply the pre-trained models to train the new data.

Step7: Classify the test data and predict the outcome.

The proposed method is further analysis through Machine Learning models and their results are shown for indicating the betterment of the Machine Learning approaches.

Pima Indian women dataset from uci machine learning repository [22] is utilized for predicting diabetic tendency of a patient. The dataset can be formulated as collection of attributes that include several criterions for detecting diabetic tendency such as number of pregnancies, patient's age, blood pressure, insulin taken, BMI percentage, Glucose level, Diabetes Pedigree function, Skin Thickness, Outcome (Diabetic/Non-diabetic). However, the attribute 'outcome' is utilized as a target class of the prediction. The dataset is partitioned into training set and testing dataset. $80 \%$ of the dataset is allocated for the training set whereas testing dataset need $20 \%$ of the dataset. Table 1 and Table 2 are indicates the performance measure results of different Machine Learning(ML) Approach.

Table1. Performance of Classifiers Using ML

\begin{tabular}{|l|l|l|l|l|}
\hline $\begin{array}{l}\text { Performance } \\
\text { Measure Metric }\end{array}$ & Accuracy & F1-Score & $\begin{array}{l}\text { Cohen-kappa } \\
\text { Score }\end{array}$ & MSE \\
\hline $\begin{array}{l}\text { Multinomial } \\
\text { Naïve Bayes } \\
\text { Classifier }\end{array}$ & $63.64 \%$ & 0.64 & 0.17 & 0.36 \\
\hline $\begin{array}{l}\text { Multilayer } \\
\text { Perceptron } \\
\text { Classifier }\end{array}$ & $71.43 \%$ & 0.71 & 0.35 & 0.29 \\
\hline $\begin{array}{l}\text { K-Nearest } \\
\text { Neighbour } \\
\text { Classifier }\end{array}$ & $75.32 \%$ & 0.75 & 0.43 & 0.25 \\
\hline $\begin{array}{l}\text { Decision Tree } \\
\text { Classifier }\end{array}$ & $76.62 \%$ & 0.77 & 0.46 & 0.21 \\
\hline
\end{tabular}

Table2. Performance of all ensemble based Classifiers

\begin{tabular}{|l|l|l|l|l|}
\hline $\begin{array}{l}\text { Performance } \\
\text { Measure Metric }\end{array}$ & Accuracy & F1-Score & $\begin{array}{l}\text { Cohen-kappa } \\
\text { Score }\end{array}$ & MSE \\
\hline $\begin{array}{l}\text { Voting Ensemble } \\
\text { Method }\end{array}$ & $78.57 \%$ & 0.79 & 0.44 & 0.21 \\
\hline $\begin{array}{l}\text { Stacking } \\
\text { Ensemble Method }\end{array}$ & $79.87 \%$ & 0.8 & 0.51 & 0.2 \\
\hline
\end{tabular}

\section{CONCLUSIONS}

Glaucoma is a neuropathic disease. It degenerates the ganglion cells of the eyes. The erosion of the eyecup enlargement damages the optic nerve and finally causes vision loss. It is one of the greatest challenges to detect glaucoma before severe stages. In this experiment, a method is proposed to detect glaucoma automatically. The proposed method helps to detect various glaucoma automatically and precisely. The method is used here ML and Stacking Ensemble Method shows the best results. 


\section{REFERENCES}

[1] Oh, S., Park, Y., Cho, K. J., \& Kim, S. J. (2021). Explainable Machine Learning Model for Glaucoma Diagnosis and Its Interpretation. Diagnostics, 11(3), 510. https://doi.org/10.3390/diagnostics11030510

[2] An, G., Omodaka, K., Hashimoto, K., Tsuda, S., Shiga, Y., Takada, N., Kikawa, T., Yokota, H., Akiba, M., \& Nakazawa, T. (2019). Glaucoma Diagnosis with Machine Learning Based on Optical Coherence Tomography and Color Fundus Images. Journal of Healthcare Engineering, 2019, 1-9. https://doi.org/10.1155/2019/4061313

[3] Odaibo, S. G. (2020). Re: Wang et al.: Machine learning models for diagnosing glaucoma from retinal nerve fiber layer thickness maps (Ophthalmology Glaucoma. 2019;2:422-428). Ophthalmology Glaucoma, 3(3), e3. https://doi.org/10.1016/j.ogla.2020.03.002

[4] Asaoka, R., Murata, H., Hirasawa, K., Fujino, Y., Matsuura, M., Miki, A., Kanamoto, T., Ikeda, Y., Mori, K., Iwase, A., Shoji, N., Inoue, K., Yamagami, J., \&Araie, M. (2019). Using Deep Learning and Transfer Learning to Accurately Diagnose Early-Onset Glaucoma From Macular Optical Coherence Tomography Images. American Journal of Ophthalmology, 198, 136-145. https://doi.org/10.1016/j.ajo.2018.10.007

[5] Barros, D. M. S., Moura, J. C. C., Freire, C. R., Taleb, A. C., Valentim, R. A. M., \&Morais, P. S. G. (2020). Machine learning applied to retinal image processing for glaucoma detection: review and perspective. BioMedical Engineering OnLine, 19(1). https://doi.org/10.1186/s12938-020$\underline{00767-2}$

[6] Thomas, P. B. M., Chan, T., Nixon, T., Muthusamy, B., \& White, A. (2019). Feasibility of simple machine learning approaches to support detection of non-glaucomatous visual fields in future automated glaucoma clinics. Eye, 33(7), 1133-1139. https://doi.org/10.1038/s41433-019$\underline{0386-2}$

[7] Lee, S. D., Lee, J. H., Choi, Y. G., You, H. C., Kang, J. H., \& Jun, C. H. (2019). Machine learning models based on the dimensionality reduction of standard automated perimetry data for glaucoma diagnosis. Artificial Intelligence in Medicine, 94, 110-116. https://doi.org/10.1016/j.artmed.2019.02.006

[8] Renukalatha, S., \& Suresh, K. V. (2019). CLASSIFICATION OF GLAUCOMA USING SIMPLIFIED-MULTICLASS SUPPORT VECTOR MACHINE. Biomedical Engineering: $\begin{array}{llll}\text { Applications, } \quad \text { Basis } \quad \text { and } & \text { Communications, } & 31(05), & \end{array}$ https://doi.org/10.4015/s101623721950039x

[9] Maetschke, S., Antony, B., Ishikawa, H., Wollstein, G., Schuman, J., \&Garnavi, R. (2019). A feature agnostic approach for glaucoma detection in OCT volumes. PLOS ONE, 14(7), e0219126. https://doi.org/10.1371/journal.pone.0219126

[10] Shabbir, A., Rasheed, A., Shehraz, H., Saleem, A., Zafar, B., Sajid, M., Ali, N., Hanif Dar, S., \& Shehryar, T. (2021). Detection of glaucoma using retinal fundus images: A comprehensive review. Mathematical Biosciences and Engineering, 18(3), 2033-2076. https://doi.org/10.3934/mbe.2021106

[11] Mursch-Edlmayr, A. S., Ng, W. S., Diniz-Filho, A., Sousa, D. C., Arnold, L., Schlenker, M. B., Duenas-Angeles, K., Keane, P. A., Crowston, J. G., \& Jayaram, H. (2020). Artificial Intelligence Algorithms to Diagnose Glaucoma and Detect Glaucoma Progression: Translation to Clinical Practice. Translational vision science \& technology, 9(2), 55. https://doi.org/10.1167/tvst.9.2.55

[12] Diagram of the Eye: Phoenix. Associated Retina Consultants. (2017, July 2). https://associatedretinaconsultants.com/diagram-of-the-eye/ 
[13] Managing Glaucoma: Best Practices for Enhancing Patient Adherence. (n.d.). https://www.powerpak.com/customcourses/107641/lesson.asp

[14] Glaucoma. (2003, May 19). WebMD. https://www.webmd.com/eye-health/glaucoma-eyes

[15] Diabetes and Eye Care: What to Watch For. (2011, July 5). WebMD. https://www.webmd.com/diabetes/diabetes-eye-care

[16] A., B., R., B., A., M., J., H., \& G., M. (2013). Robust vessel segmentation in fundus images. International Journal of Biomedical Imaging, 2013, 1-22. http://www.embase.com/search/results?subaction=viewrecord\&from=export\&id=L372348681 \%0Ahttp://dx.doi.org/10.1155/2013/154860

[17] Diaz-Pinto, A., Morales, S., Naranjo, V., Köhler, T., Mossi, J. M., \&Navea, A. (2019). CNNs for automatic glaucoma assessment using fundus images: An extensive validation. BioMedical Engineering Online, 18(1), 1-19. https://doi.org/10.1186/s12938-019-0649-y

[18] Hassan, M. U. (2021, February 24). VGG16 - Convolutional Network for Classification and Detection. Neurohive. https://neurohive.io/en/popular-networks/vgg16/

[19] Mateen, M., Wen, J., Nasrullah, Song, S., \& Huang, Z. (2019). Fundus image classification using VGG-19 architecture with PCA and SVD. Symmetry, 11(1). https://doi.org/10.3390/sym11010001

[20] Szegedy, C., Wei Liu, Yangqing Jia, Sermanet, P., Reed, S., Anguelov, D., Rabinovich, A. (2015). Going deeper with convolutions. 2015 IEEE Conference on Computer Vision and Pattern Recognition (CVPR). doi:10.1109/cvpr.2015.7298594

[21] Yao, N., Ni, F., Wang, Z., Luo, J., Sung, W. K., Luo, C., \& Li, G. (2021). L2MXception: an improved Xception network for classification of peach diseases. Plant Methods, 17(1), 1-13. https://doi.org/10.1186/s13007-021-00736-3

[22] Michael Kahn, St. Louis. UCI Machine Learning Repository [http://archive.ics.uci.edu/ml]. Irvine, CA: University of California, School of Information and Computer Science. 